\title{
TESTING EFFICIENCY OF INDEX NUMBERS AND ITS ROLE IN ELEMINATING THE INFLATION
}

\author{
HUSSEIN, H. F. M. and I. M. M. JABR
}

Econ. \& Enviro. Evaluation Res. Sec., Cent. Lab. For Design and Stat. Analysis Res., ARC, Egypt.

(Manuscript received 23 November 2017)

\begin{abstract}
$\mathrm{T}$ he study aims to estimate the aggregate index number for grain group prices in the production quantities during the period (2006-2011) as the base period and the period (2011-2015) as a comparative period. The results showed that the index lasper number was about $53 \%$ that of Bashy was about $54.3 \%$ that of Druobshy and Baly was about $35.75 \%$, Fisher's index number was about $52.55 \%$ and the Marshall-Edgorth index number was about 53.59\%. The results showed that Fisher's index number is the only one that achieves the condition of reflection in the coefficient and the reflection in the time, so it is characterized by high accuracy and quality of the estimate. The study also showed that the decline in the real value of money is the most important reason for the high level of inflation in the agricultural prices of grain crops. Therefore, the study recommends estimating $s$ index numbers for prices of agricultural crops in Egypt should be based on Fisher's index number. The study also recommends adjusting the current prices of agricultural crops at constant prices to eliminate inflation, taking into consideration changing tastes, unequal changes in price levels for all members of the society, and changing the speed of money circulation due to changing purchasing habits of individuals. The study also recommends using a new year as the base year and not relying on an old year and encourage the governmental corporations to produce accurate records.
\end{abstract}




\title{
الكفاءة النسبية للأرقام القياسية ودورها في تقدير قيم التضخم
}

\author{
هبة فهمي محمد حسين ، إيناس ممدوح محمود جبر \\ قسم بحوث التقبيم الاقتصادي و البيئي- المعدل المركزي لبحوث التصميم والتحليل الإحصائي \\ مركز البحوث الزراعية

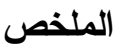

تهدف الدراسة لتقدير الكفاءة النسبية لبعض الأرقام القياسية ودور ها في تقدير قيم التضخم

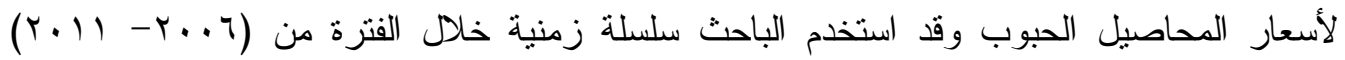

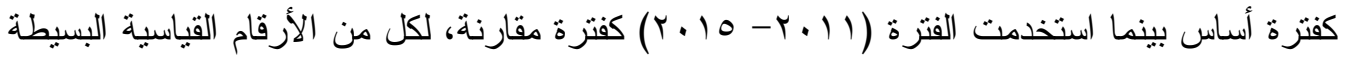
و الأرقام القياسية التجميعية وتقدير الأرقم القياسية لكل من لاسبير وباثىى ودورشى وبإلي وفيشر

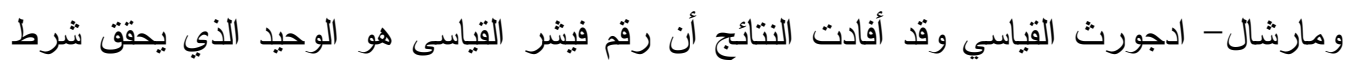
الانعكاس في المعامل و الانعكاس في الزمن لذا فهو ينسم بارتفاع دقة وجودة التقدير ، تبين أن رقم

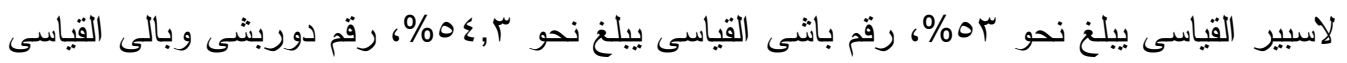

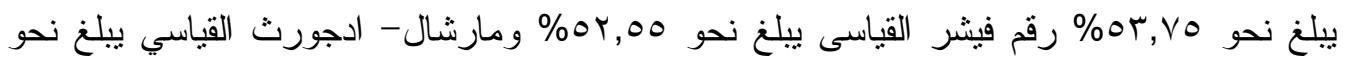
09,09، ومن ثم توصى الدر اسة بالاعتماد على رقم فيشر القياسى في تقدير الأرقام القياسية لأسعار

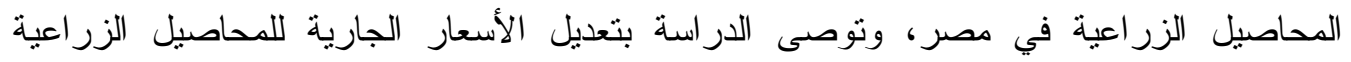
بالأسعار الثابتة لاستبعاد التضخم ومر اعاة تغير الأذو اق و عدم تساوى التغير ات في مستويات الأسعار

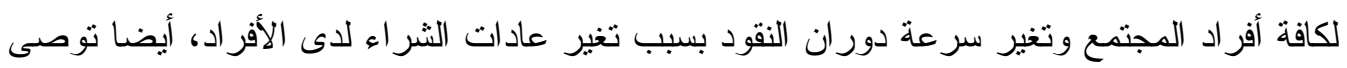

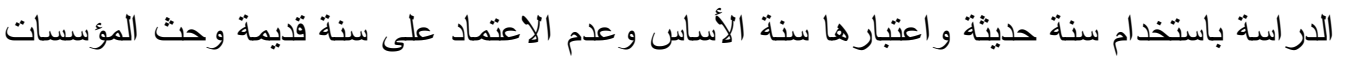
الحكومية على إصدار أرقام قياسية تتسم بالدقة.

\section{مقدمة}

يعكف الباحثون ومتخذو القرار علي رصد التغيرات التي تطرأ على أسعار وكميات هذه المحاصيل التي يتم التعامل بها في الأسواق من خلال الأرقام القياسية والتي تعتبر أداة إحصائية

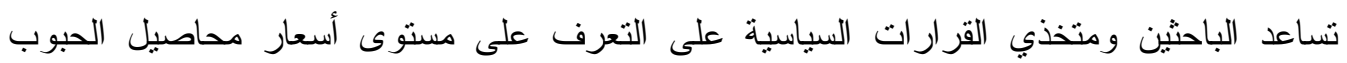

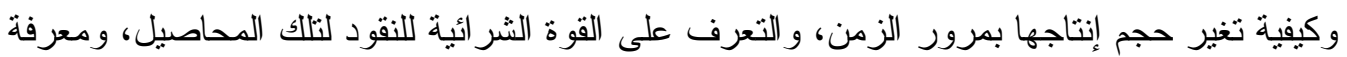

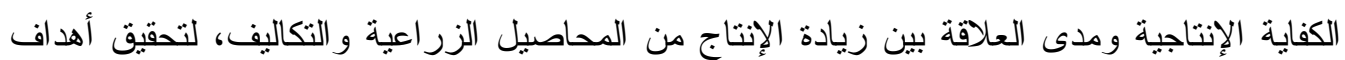
عملية التتمية الاقتصادية وتلبية احتياجات الأسرة المعيثية، يعد الرقم القياسى أداة إحصائية مهمة لقياس مقدار التغير في الظواهر الاقتصادية خلال فتزتين زمنيتين أو مكانين تسمى الأولى فترة

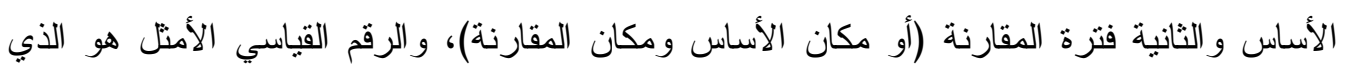
يحقق اختباري الانعكاس الزمني في الأساس و الانعكاس في المعامل. 


\section{مشكلة البحث}

يؤدى إغفال الباحثين ومتخذي القرار لأهمية الأرقام القياسية للمحاصيل الزر اعية وتطبيقاتها

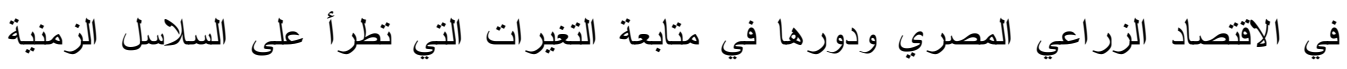
لأسعار و إنتاج محاصيل الحبوب وأهميتها في تقسير عامل التضخم، وما بنطوي علي استخدام الأسعار الجارية عند تفسير تطور الأسعار المزرعية إلى نتائج سلبية تؤئز سلبياً على الحياة الاقتصادية والاجتماعية للمستهلك، فضلا عن الآثار السلبية المترنبة على استخدامها في التحليلات الإحصائية و الاقتصادية ومن ثم انخفاض مستوى الثقة في التتبؤ بالمعادلات الإحصائية لمعدلات النمو

\section{يهرف البحث إلى}

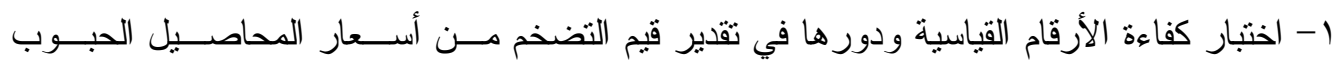

r- بيان أهميتها في التتبؤ وتصحيح معدلات نمو الأسعار المزرعية لمحاصيل الحبوب الغذائية.

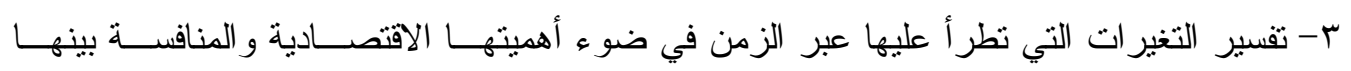
و إجمالي الناتج منها. ع - بيان تطبيق وأهمية الأرقام القياسية في تقدير التضخم و المقارنة فيما بينها لتحديد أفضل أســلوب لتقدير الرقم القياسي لهذه المحاصيل و البيانات المطلوبة للنقدير طبقا للكفاءة النسبية لها.

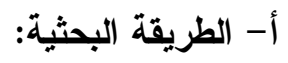
استخدم الباحث بعض أنماط من طرق تقدير الأرقام القياسية كالأتي: 1- تقدير الأرقام القياسية لمحاصيل الحبوب بطريقة الأرقام القياسية البسيطة والأرقام القياسية التجميعية. ז- تقدير الأرقم القياسية لكل من لاسبير وباثى ودورشى وبإلي فيشر ومارشال - ادجورث القياسي وتقدير الأرقام القياسية النسبية. r- إجر اء اختبار ات الانعكاس في الزمن وانفيلة اختبار الانعكاس في المعامل لبيان جودة الأرقام القياسية لتوضيح أي من هذه يعبر عن حقيقة التغير في السلاسل الزمنية لأسعار و إنتاج محاصيل الحبوب. ب- مصادر البيانات:

استعانت الدراسة ببعض الأساليب الإحصائية منل أسلوب تحليل الانحدار ، وذلك بالاعتماد

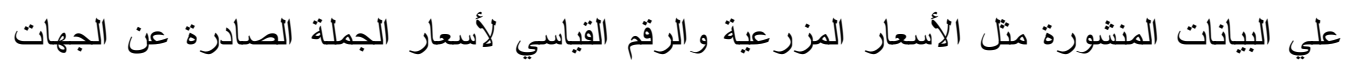

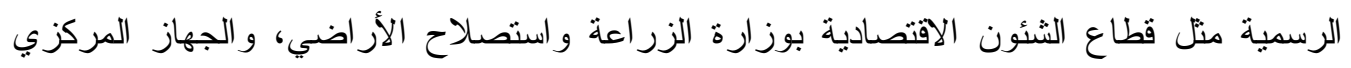

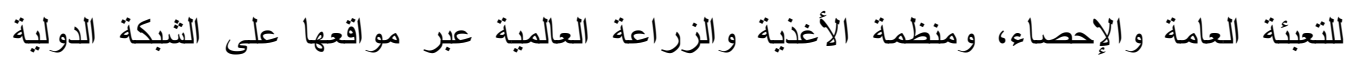

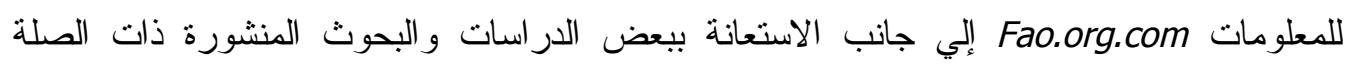
بموضوع الدر اسة. 


\section{الأساس النظري للأرقام القياسية}

الرقم القياسي هو أداة إحصائية لقياس التغير النسبي في ظاهرة أو مجموعة من الظواهر

خلال فترة زمنية سواء كانت الظاهرة كمية أو قيمة أو أجراً، ويطلق على الفترة التي ننسب إليها فترة الأساس و الفترة التي ننسبها فترة المقارنة، ويمكن من خلال الرقم القياسي التعرف على مستوى أسعار مجموعة من السلع، أو كيفية تغير حجم إنتاجها بمرور الزمن، ولذلك يمكن بواسطته مقارنة

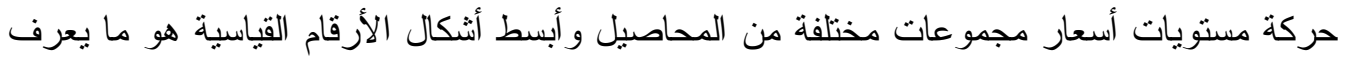
بمنسوب السعر وهو عبارة عن قسمة قيمة الظاهرة في فترة معينة أو مكان معين على قيمتها في فترة أخرى أو مكان أخر كذللك بالنسبة للمكان المنسوب إليه مكان الأساس و المكان الذي ننسبه مكان المقارنة، فإذا كان الرقم القياسي أقل من .1\% فذللك يدل على النقصان في الظاهرة، و إذا كان الرقم القياسي أكبر من . . 1\% فذلك يدل على الزيادة في الظاهرة، ويجب أن تتسم فترة الأساس بالاستقرار وتكون خالية من التغيرات غير المنتظمة والعرضية ومن أي تشوهات يمكن أن تطر أ على الظو اهر

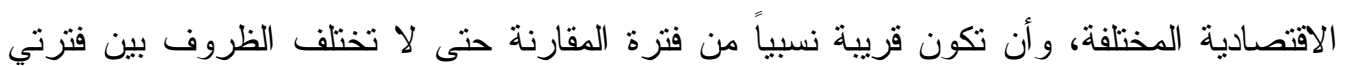

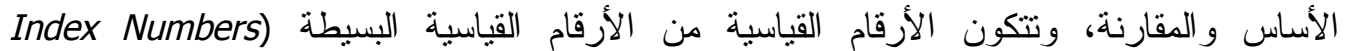
(Simple)، الأرقام القياسية التجميعية (Aggregative Index Numbers)، الأرقام القياسية النسبية (Relative Index Numbers)

\section{طرق تقدير الأرقام القياسية}

ا 1 - الرقم القياسي البسيط Simple Index

الرقم القياسي البسيط للأسعار أو الكميات هو عبارة عن حاصل قسمة مجموع أسعار

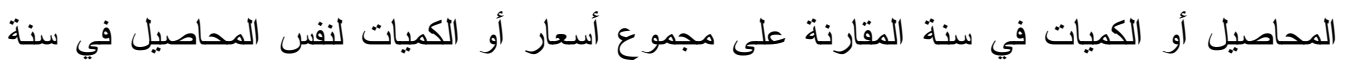

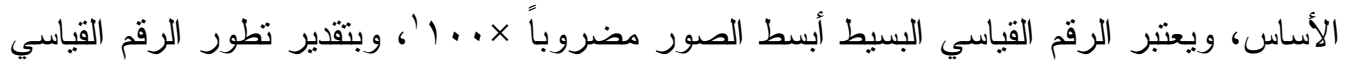

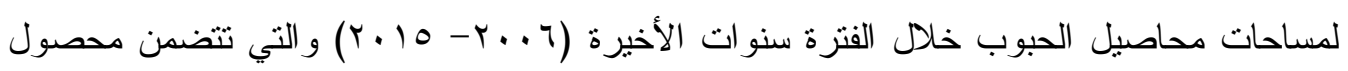

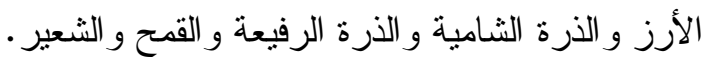

$$
\text { IN }=\frac{A_{1}}{A_{0}} \times 100
$$

= الرقم القياسي البسيط للمساحة المنزرعة لمحاصيل الحبوب. IN = $A_{0}$ 
الرقم القياسي لتطور إنتاج مجموعة محاصيل الحبوب:

يتم تقدير الأرقام القياسية لإنتاج مجموعة محاصيل الحبوب لتطور الأرقام القياســــة خــلدل

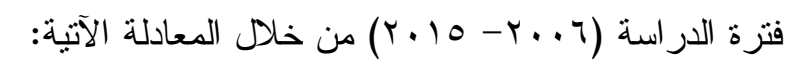

$$
I N=\frac{Q_{1}}{Q_{0}} \times 100
$$

= $=Q_{1}$

= $=Q_{0}$

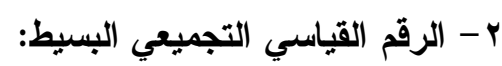

يتم حساب الرقم القياسى التجميعي البسيط بالنسبة للأسعار و الكميات وذلك من خلال :

$I N=\frac{\sum p_{1}}{\sum p_{0}} \times 100$

$I N=\frac{\sum Q_{1}}{\sum Q_{0}} \times 100$

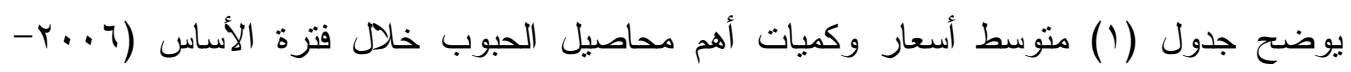

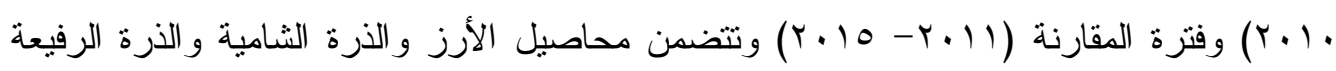
و القمح و الثعبر ، وقد تبين بالنسبة لأسعار المحاصيل موضو ع الدراسة الدة ارتفاع أسعار محاصيل الأرز

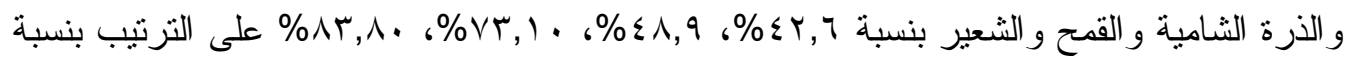

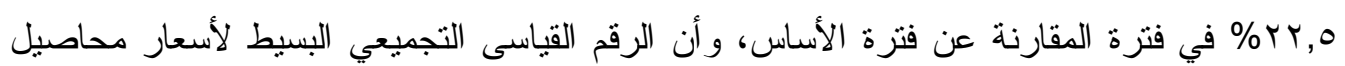

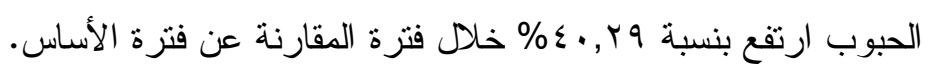

بالنسبة لكميات إنتاج أهم محاصيل الحبوب فينتين زيادة إنتاج محاصيل الذرة الثامية و القمح بنسبة

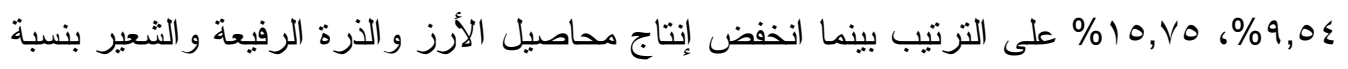

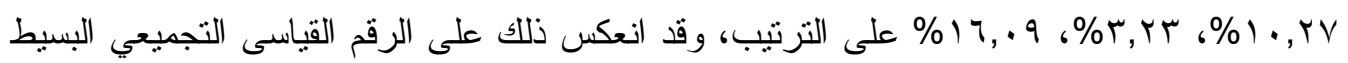

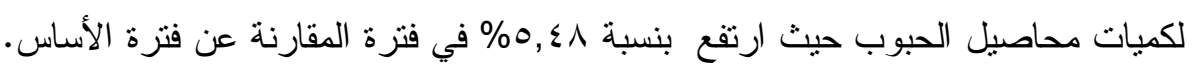

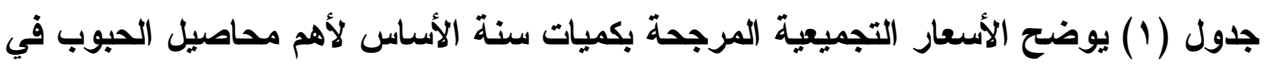

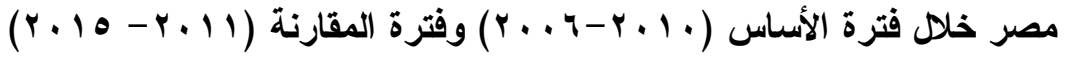

\begin{tabular}{|c|c|c|c|c|c|c|}
\hline القياسي & \multicolumn{2}{|c|}{ كميات المحاصيل بالأف } & الرقم القياسي & \multicolumn{2}{|c|}{ أسعار المحاصيل بالجنيه } & \multirow[b]{2}{*}{ المحاصيل } \\
\hline$\%$ & $\begin{array}{c}\text { فترة } \\
\text { المقارنة } \\
Q_{1}\end{array}$ & $\begin{array}{c}\text { فترس الأساس } \\
Q_{0}\end{array}$ & $\%$ & المقارنة & الأسساس & \\
\hline$\wedge \vee, \vee \vee$ & 0011,0 & $71 r 9,7$ & $1 \leqslant Y, 7$ & $r . q$. & $1 \leq 70$ & الأرز \\
\hline $1.9,0 \leqslant$ & $7 \wedge 99, r$ & $\because r 9 \Lambda, \varepsilon$ & $1 \leqslant \Lambda, 9$ & riA. & $1 \leqslant 7 \leqslant$ & الذرة الثشامية \\
\hline $97, V \vee$ & VYI & $\vee 97, \vee$ & $V r, O$ & $170 \mathrm{r}$ & $r r \leqslant 9$ & الذرة الرفيعة \\
\hline 110,10 & $91 \cdot r, V$ & VAT & IVT,1 & rNoq & 1704 & القمح \\
\hline$\wedge r, q 1$ & $\| r, r$ & $1 r r, q$ & $1 \wedge r, \Lambda$ & rTAY & $1 \wedge \varepsilon$. & الثعير \\
\hline $1 \cdot 0, \varepsilon \wedge$ & TIRTT, & rrraq, & $1 \varepsilon \cdot, r$ & Tr/Tr & NTV. & المجموع \\
\hline
\end{tabular}

حسبت من بيانات جدول ( ) وجدول (؟) بالملسحق. 


\section{r- الرقم القياسي التجميعي المرجح:}

يحسب الرقم القياسي التجميعي بترجيح أسعار كل المحاصيل بمعامل مناسب لأهمية المحاصيل المختلفة كل على حسب أهميته، وغالباً ما يتم الترجيح أما بالكمية أو قيمة السلعة خلال فترة الأساس لهاس أو فترة المقارنة وذلك بهدف إبر از الأهمية النسبية، توجد عدة طرق لتقدير الرقم القياسي التجميعي المرجح أهمها و أكثر ها شيو عاً (رقم لاسبير القياسي) و الذي يحسب كما يلي:

$$
L=\frac{\sum P_{1} Q_{0}}{\sum P_{0} Q_{0}} \times 100
$$

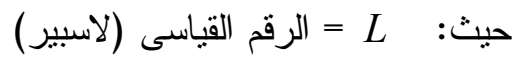

مجموع أسعار المحاصيل مرجح بكميات في سنة الأساس.

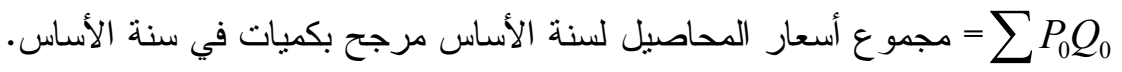

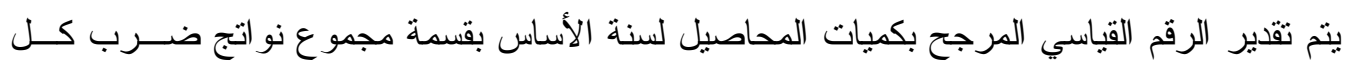

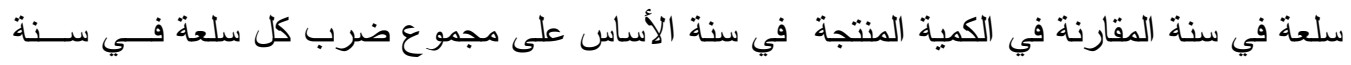

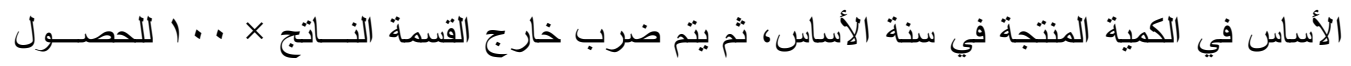

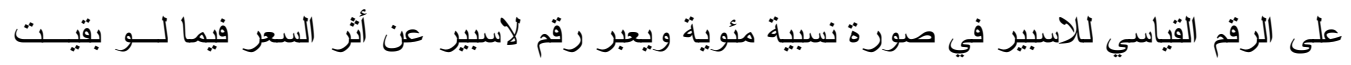
الكمية المشتر اه على نفس المستوى في سنة الأساس.

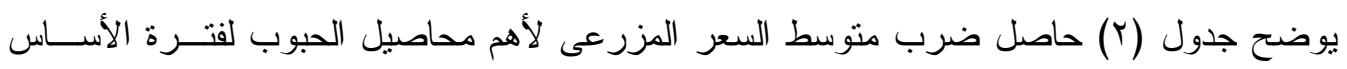

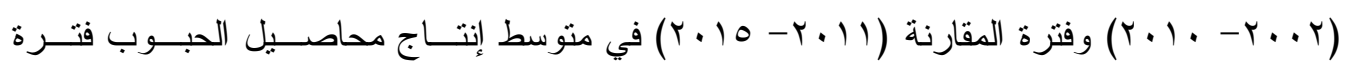

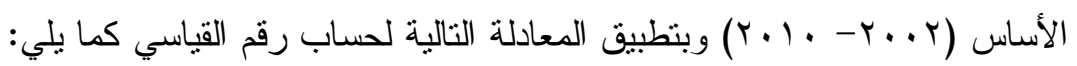
$L=\frac{\sum P_{1} Q_{0}}{\sum P_{0} Q_{0}} \times 100=1.528 \times 100=153 \%$

بتقدير الرقم القياسى التجميعي المرجح للاسبير يتبين أنه يبلغ به \% وهو مـــا يعنـى أن

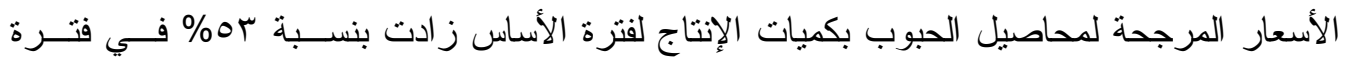

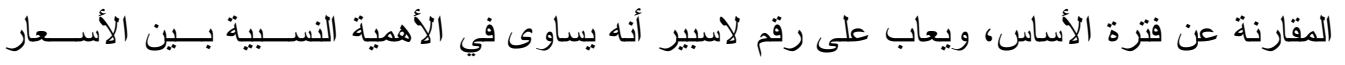

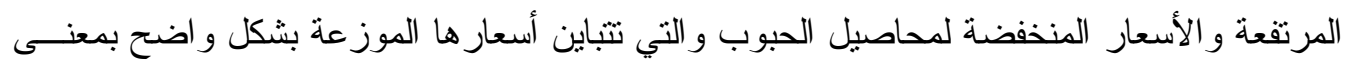

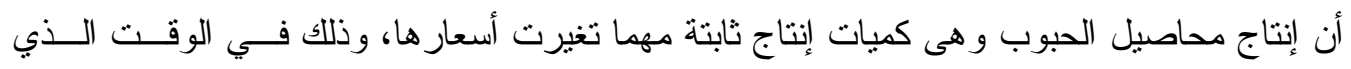

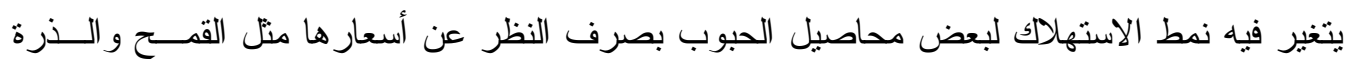
الثامية وخاصة إذا اخذ في الاعتبار تعدد وتباين العو امل المؤثرة على الكميات المنتجـــة و الأســعار المزرعية لمحاصيل الحبوب.

$$
\text { ع - تقدير الرقم القياسي بطريقة باشسى: }
$$

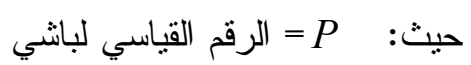
= $=P_{1} Q_{1}$ 
= مجموع أسعار المحاصيل لسنة الأساس مرجح بكميات في سنة المقارنة.

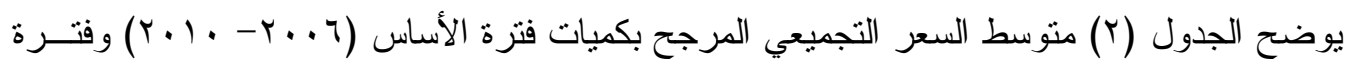

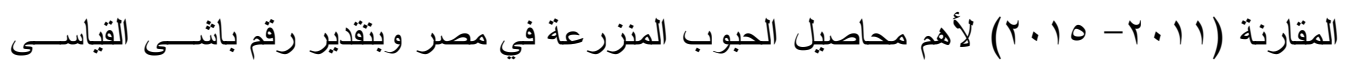
من المعادلة السابقة تبين أن: $P=\frac{\sum P_{1} Q_{1}}{\sum P_{0} Q_{1}} \times 100=1.523 \times 100=154.3 \%$

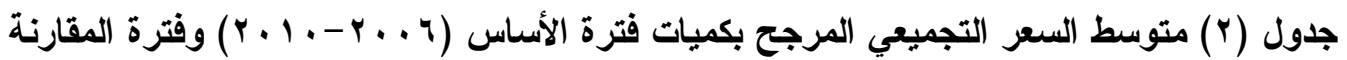

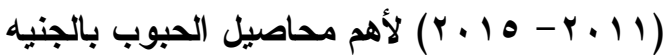

\begin{tabular}{|c|c|c|c|c|}
\hline \multicolumn{2}{|c|}{ الأسعار التجميعية المرجحة بكميات سنة المقارنة } & \multicolumn{2}{|c|}{ الأسعار التجميعية المرجحة بكميات سنة الأسساس } & \multirow[b]{2}{*}{ 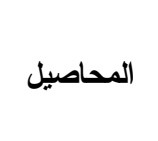 } \\
\hline$P_{0} Q_{1}$ & $P_{1} Q_{1}$ & $P_{0} Q_{0}$ & $P_{1} Q_{0}$ & \\
\hline$\Lambda \cdot v \leqslant r q r$ & $110191 \ldots$ & ᄉ99 0 Or. & ITATIVVT & الأرز \\
\hline $1.1 .0 \mathrm{Or}$ & $10.5 \cdot \leq 11$ & qrr.ANA & $1 \pi V T .001$ & 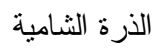 \\
\hline $\mid v r \leqslant 11 \wedge$ & ITVTVqs & $\mid \vee 91911$ & ITITY & 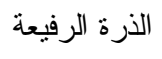 \\
\hline $10 . r V V \leq r$ & $r T \cdot r \leqslant V T l$ & Irq9r. & $r Y \leq \Lambda \leq \varepsilon \leq \varepsilon$ & القمح \\
\hline r.TVAI & $r \Lambda \ldots \vee r$ & r $\left\{T \leqslant T_{0}\right.$ & 纟or901 & الشعبر \\
\hline roloroth & $0 \leq r r \wedge I r q$ & MrT $\leqslant 0 \wedge 10$ & 0.117 .49 & 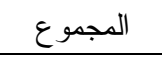 \\
\hline
\end{tabular}

المصدر : حسبت وجمعت من جدول (r) بالملحق.

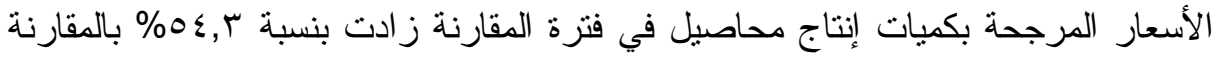
بقترة الأساس وهو ما يشير إلى أن نمط الاستهلاك لمحاصيل الحبوب في مصر يتغير بتغير الزمن فئن

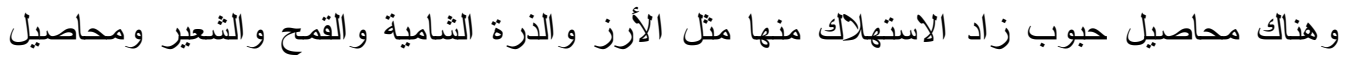

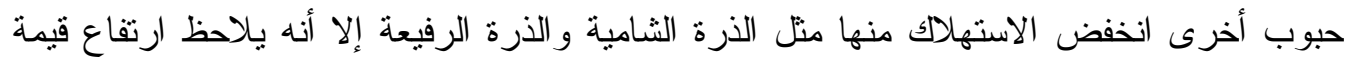
محاصيل الحبوب المنتجة في مصر بصفة عامة في فترة المقارنة عن فترة الأساس، ويعاب على رقم باثىى القياسي أنه يعتمد في تقديره على استخدام أوزان ترجيحية بصفة مستمرة مما يحتاج إلى جهد

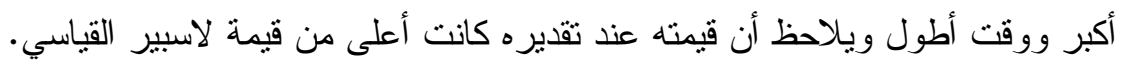
ه - تقدير الرقم القياسي لدوربشى وبالي (المتوسط الحسابي لرقم لاسبير وياشى):

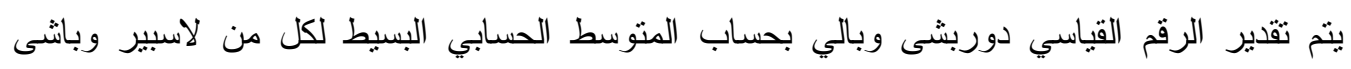
القياسي، أي أن رقم دوربشى وبالي القياسي ما هو إلا المتوسط الحسابي البسيط لكل من الرقم

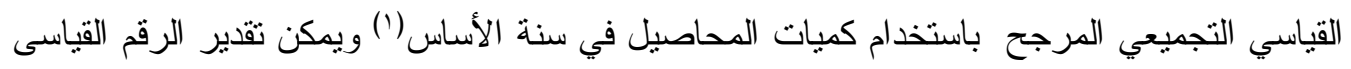

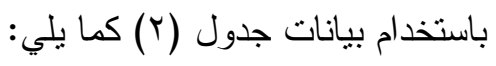




$$
D M=\frac{\frac{\sum p_{1} Q_{0}}{\sum p_{0} Q_{0}}+\frac{\sum p_{1} Q_{1}}{\sum p_{0} Q_{1}}}{2} \times 100=\frac{1.528+1.543}{2} \times 100=153.75 \%
$$

أي أن الرقم القياسي دوربشى وبالي المحسوب لمجموعة محاصيل الحبوب التي تضم الأرز

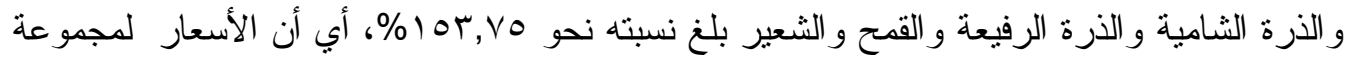

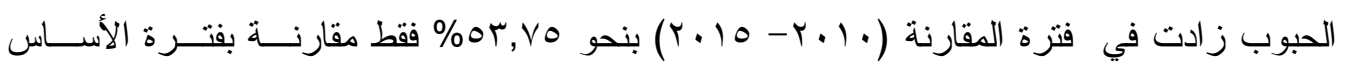

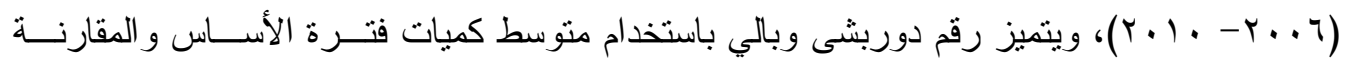
ليحصل علي رقم أفضل من رقمي (لاسبير وبانشى). צ- تقدير الرقم القياسي لفيشر: هو الرقم القياسي الأمتل ويعبر عنه بأنه الوسط الهنسي للأرقام القياسية التجميعية المرجحة لكل من

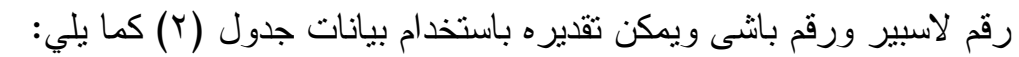
$F=\sqrt{\frac{\sum p_{1} Q_{0}}{\sum p_{0} Q_{0}} \times \frac{\sum p_{1} Q_{1}}{\sum p_{0} Q_{1}}} \times 100=\sqrt{1.528 \times 1.523} \times 100=152.55 \%$

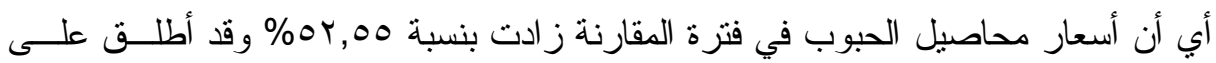

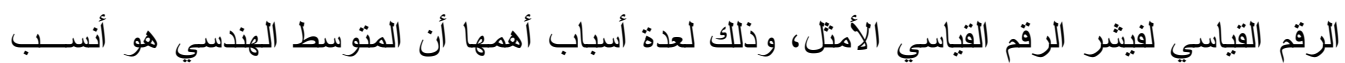

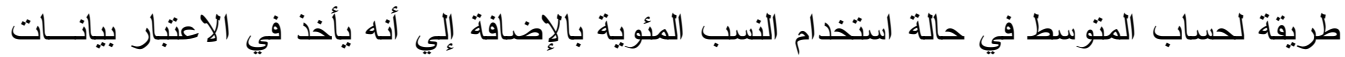
كميات فترة الأساس وفترة المقارنة.

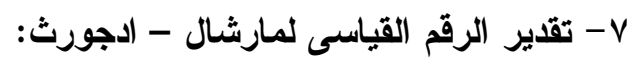

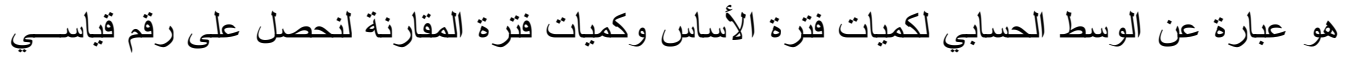

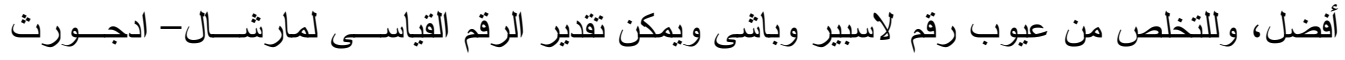
$M I=\frac{\sum\left(P_{1}\left(Q_{0}+Q_{1}\right)\right)}{\sum\left(P_{0}\left(Q_{0}+Q_{1}\right)\right)}=\frac{50816029+54238139}{33245885+35153568} \times 100=153.59 \%$

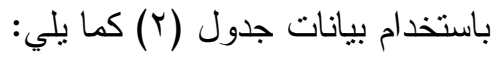

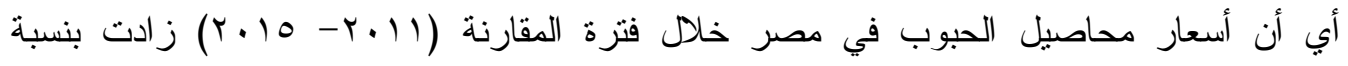

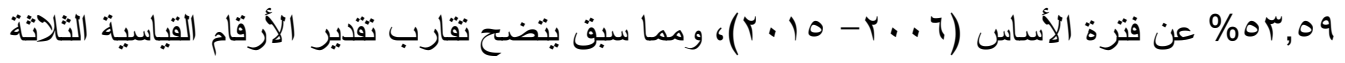

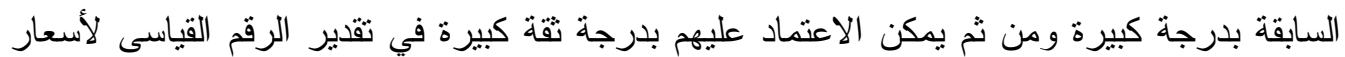

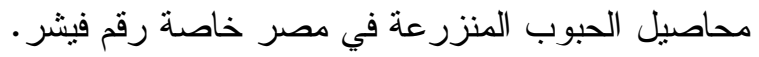
اختبار الكفاءة النسبية للأرقام القياسية:

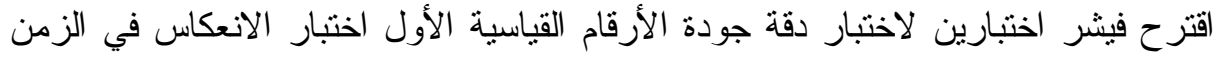

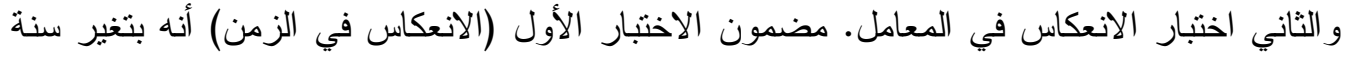
الأساس إلى سنة المقارنة وسنة المقارنة إلى سنة الأساس فان الرقم القياسى × البديل الزمني له له 
يساوى واحد(') وبتطبيق هذا الاختبار على بيانات أسعار وكميات إنتاج أهم محاصيل الحبوب خلال

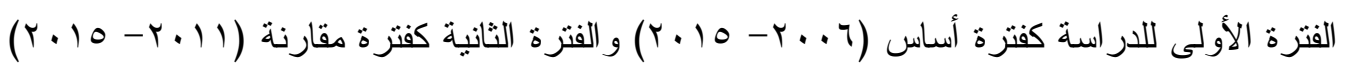
كما يلي: أولاً: اختبار الانعكاس في الزمن:

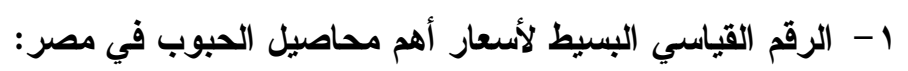

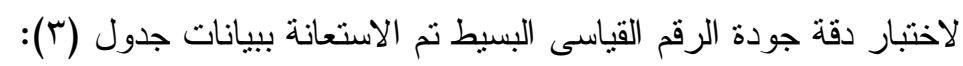

$\frac{\sum P_{1}}{\sum P_{0}} \times \frac{\sum P_{0}}{\sum P_{1}}=\frac{12163}{8670} \times \frac{8670}{12163}=1$

أي أن الرقم القياسى البسيط لأسعار محاصيل الحبوب المقدرة خلال فترة الدراسة يحقق شرط الانعكاس الزمني وهو ما يشير إلى الدقة وارتفاع جودة الأرقام القياسية المقدة لمحاصيل الحبوب موضوع الدر اسة. r - الرقم القياسي التجميعي المرجح بكميات سنة الأساس (رقم لاسبير القياسى):

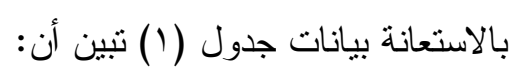

$\frac{\sum P_{1} Q_{0}}{\sum P_{0} Q_{0}} \times \frac{\sum P_{0} Q_{1}}{\sum P_{1} Q_{1}}=1.528 \times 0.65 \neq 1$

أي أن الرقم القياسى التجميعي المرجح بكميات فترة الأساس لأسعار محاصيل الحبوب

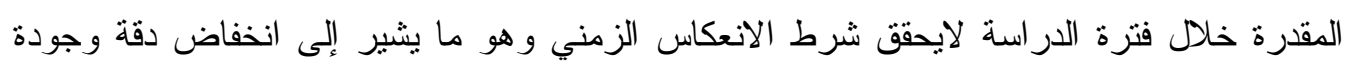
الأرقام القياسية المقدرة لمحاصيل الحبوب موضع الدرة اسة باستخدام رقم لاسبير القياسي. r- الرقم القياسي التجميعي المرجح بكميات سنة المقارنة (رقم باشى القياسي):

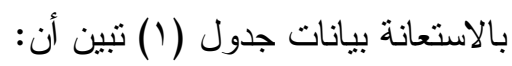

$\frac{\sum P_{1} Q_{1}}{\sum P_{0} Q_{1}} \times \frac{\sum P_{0} Q_{0}}{\sum P_{1} Q_{0}}=1.543 \times 0.648 \neq 1$

آي أن الرقم القياسى التجميعي المرجح بكميات سنة المقارنة لأسعار محاصيل الحبوب المقدرة خلال فترة الدراسة لا يحقق شرط الانعكاس الزمن وهو ما يثير إلى دقة الأرقام القياسية المقدرة لمحاصيل الحبوب موضوع الدر اسة باستخدام الرقم باشىى القياسى.

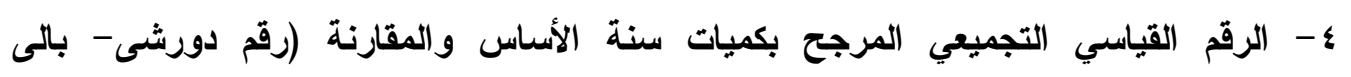
(القياسى): بالاستعانة بيانات جدول (r) تبين أن: $\frac{\frac{\sum P_{1} Q_{0}}{\sum P_{0} Q_{0}}+\frac{\sum P_{1} Q_{1}}{\sum P_{0} Q_{1}}}{2} \times \frac{\frac{\sum P_{0} Q_{1}}{\sum P_{1} Q_{1}}+\frac{\sum P_{0} Q_{0}}{\sum P_{1} Q_{0}}}{2} \neq 1$

$1.536 \times 0.65 \neq 1$

ا مو ارى د.شبيجيل ( •99 ())، "الإحصاء"، سلسلة ملخصات شوم، الدار الدولية للنشر و النوزيع، القاهرة. 
أي أن الرقم القياسى التجميعي المرجح بكميات سنة الأساس والمقارنة لأسعار محاصيل الحبوب المقدرة خلال فترة الدراسة لا يحقق شرط الانعكاس الزمني وهو ما يشير إلى انخفاض دقة لإنة

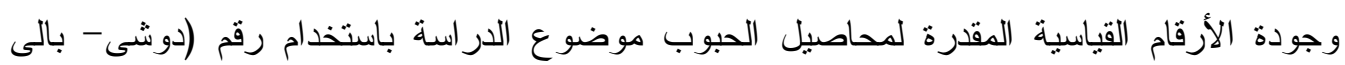
(القياسى). هـ - الرقم القياسي التجميعي المرجح والمحسوب على أساس المتوسط الهنسي لكل من رقم لاسبير ورقم باشى معاً (رقم فيشر القياسي).

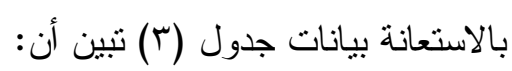
$\sqrt{\frac{\sum P_{1} Q_{0}}{\sum P_{0} Q_{0}} \times \frac{\sum P_{1} Q_{1}}{\sum P_{0} Q_{1}}} \times \sqrt{\frac{\sum P_{0} Q_{1}}{\sum P_{1} Q_{1}} \times \frac{\sum P_{0} Q_{0}}{\sum P_{1} Q_{0}}}=\sqrt{2.358} \times \sqrt{0.424}=1$ أي أن الرقم القياسى التجميعي والمحسوب على أساس المنوسط الهندسي لكل من رقم

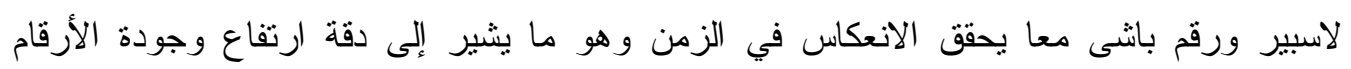

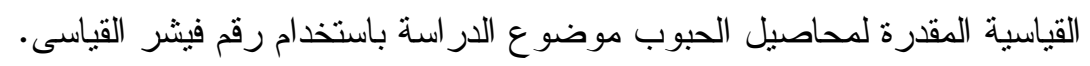

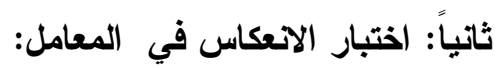
مضمون هذا الاختبار أنه بتغير الكميات إلي أسعار والأسعار إلي كميات عندئذ فان الرقم القياسي ×

$$
\text { البديل المعاملى = منسوب القيمة. }
$$$$
\frac{\sum P_{1}}{\sum P_{0}} \times \frac{\sum Q_{1}}{\sum Q_{0}} \neq \frac{\sum P_{1} Q_{1}}{\sum P_{0} Q_{0}}
$$

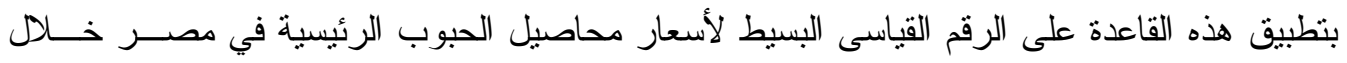

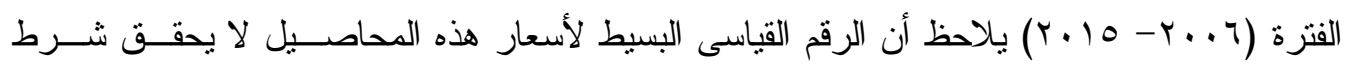

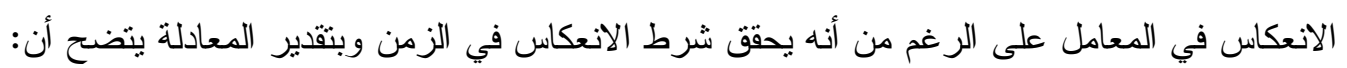
$\frac{\sum P_{1}}{\sum P_{0}} \times \frac{\sum Q_{1}}{\sum Q_{0}}=1.403 \times 1.055=1.48$ $\frac{\sum P_{1} Q_{1}}{\sum P_{0} Q_{0}}=1.63$ $1.48 \neq 1.63$

ץ- الرقم القياسي التجميعي المرجع بكميات فترة الأسساس (رقم لاسبير القياسى): $\frac{\sum P_{1} Q_{0}}{\sum P_{0} Q_{0}} \times \frac{\sum Q_{1} P_{0}}{\sum Q_{0} P_{0}} \neq \frac{\sum P_{1} Q_{1}}{\sum P_{0} Q_{0}}$

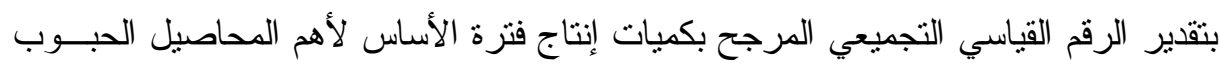

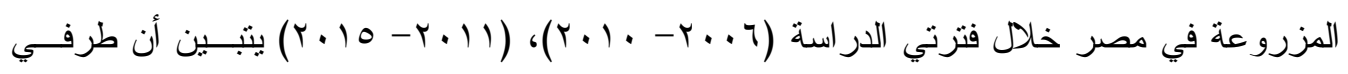
المعادلة متساويان مما يثير إلى أن رقم لاسبير القياسى يحقق شرط الانعكاس في المعامل كما أنه لا لا 
يحقق شرط الانعكاس في الزمن مما يدل على انخفاض دقة وجودة رقم لاسـبير القياســي وبتقــير

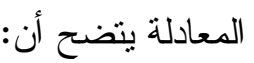

$\frac{\sum P_{1} Q_{0}}{\sum P_{0} Q_{0}} \times \frac{\sum Q_{1} P_{0}}{\sum Q_{0} P_{0}}=1.62$

$\frac{\sum P_{1} Q_{1}}{\sum P_{0} Q_{0}}=1.63$

$1.63=1.63$

r- الرقم القياسي التجميعي المرجح بكميات فترة المقارنة (رقم باشى القياسى): $\frac{\sum P_{1} Q_{1}}{\sum P_{0} Q_{1}} \times \frac{\sum Q_{1} P_{1}}{\sum Q_{0} P_{1}} \neq \frac{\sum P_{1} Q_{1}}{\sum P_{0} Q_{0}}$

$\frac{\sum P_{1} Q_{1}}{\sum P_{0} Q_{1}} \times \frac{\sum Q_{1} P_{1}}{\sum Q_{0} P_{1}}=1.65$

$\frac{\sum P_{1} Q_{1}}{\sum P_{0} Q_{0}}=1.63$

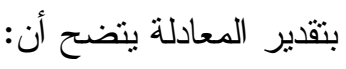

$1.65 \neq 1.63$

بتقدير الرقم القياسي التجميعي المرجح بكميات سنة المقارنة لأهم محاصيل الحبوب المزروعة في

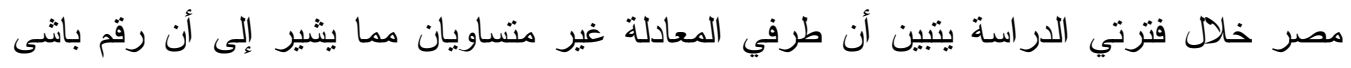

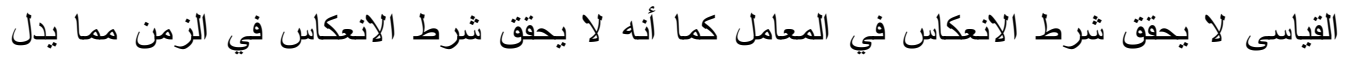

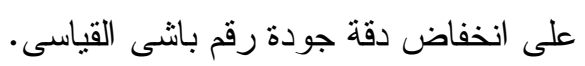
ع - الرقم القياسي التجميعي المرجح بكميات فترة الأسساس وفترة المقارنة (رقم دورشى - وبالى

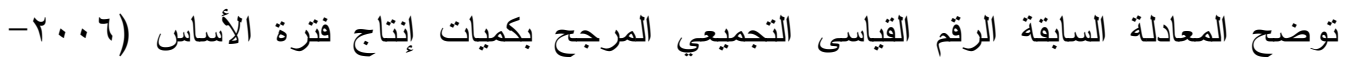

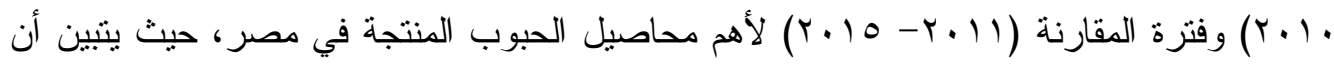

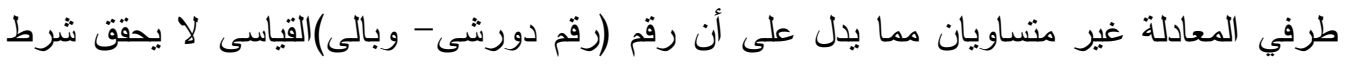
الانعكاس في الزمن مما يدل على انخفاض دقة وجودة رقم (دورشى - وبالى) القياسى. $\frac{\frac{\sum P_{1} Q_{0}}{\sum P_{0} Q_{0}} \times \frac{\sum Q_{1} P_{0}}{\sum Q_{0} P_{0}}}{2} \times \frac{\frac{\sum P_{1} Q_{1}}{\sum P_{0} Q_{1}} \times \frac{\sum Q_{1} P_{1}}{\sum Q_{0} P_{1}}}{2} \neq \frac{\sum P_{1} Q_{1}}{\sum P_{0} Q_{0}}$

$\frac{\frac{\sum P_{1} Q_{0}}{\sum P_{0} Q_{0}} \times \frac{\sum Q_{1} P_{0}}{\sum Q_{0} P_{0}}}{2} \times \frac{\frac{\sum P_{1} Q_{1}}{\sum P_{0} Q_{1}} \times \frac{\sum Q_{1} P_{1}}{\sum Q_{0} P_{1}}}{2}=1.996$

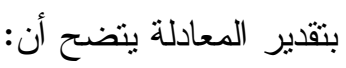
$\frac{\sum P_{1} Q_{1}}{\sum P_{0} Q_{0}}=1.63$ 
$1.996 \neq 1.63$

ه- الرقم القياسي التجميعي المرجح المحسوب على أساس المتوسط الهندسي لكل لاسبير وياشى معاً (رقم فيشر): $\sqrt{\frac{\sum P_{1} Q_{0}}{\sum P_{0} Q_{0}} \times \frac{\sum P_{1} Q_{1}}{\sum P_{0} Q_{1}}} \times \sqrt{\frac{\sum P_{1} Q_{0}}{\sum P_{0} Q_{0}} \times \frac{\sum P_{1} Q_{1}}{\sum P_{0} Q_{1}}}=\frac{\sum P_{1} Q_{1}}{\sum P_{0} Q_{0}}$

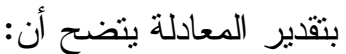

$\sqrt{\frac{\sum P_{1} Q_{0}}{\sum P_{0} Q_{0}} \times \frac{\sum P_{1} Q_{1}}{\sum P_{0} Q_{1}}} \times \sqrt{\frac{\sum P_{1} Q_{0}}{\sum P_{0} Q_{0}} \times \frac{\sum P_{1} Q_{1}}{\sum P_{0} Q_{1}}}=\sqrt{1.616} \times \sqrt{1.646}=1.63$

$\frac{\sum P_{1} Q_{1}}{\sum P_{0} Q_{0}}=1.63$

$1.63=1.63$

توضح المعادلة السابقة الرقم القياسى التجميعي المرجح المحسوب على أساس المنوسط الهندسي لكل

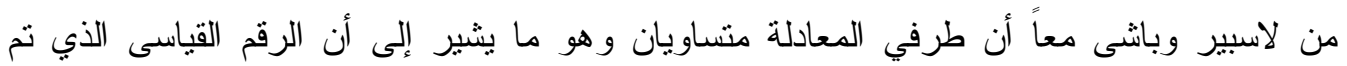

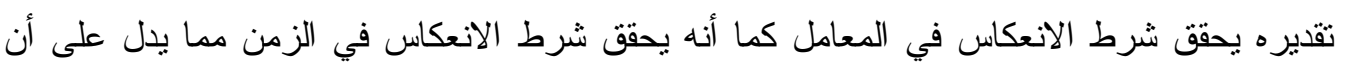

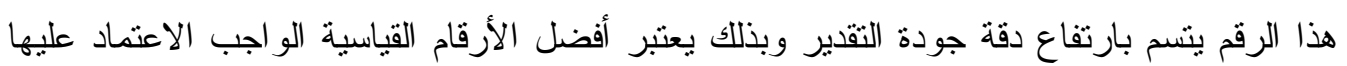

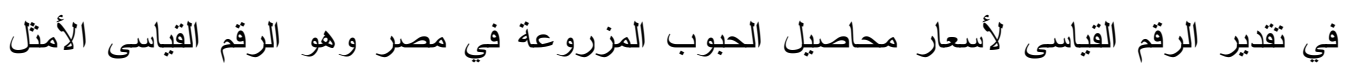
لاستخدامه في التقدير ('). يوضح شكل (1) تقدير الرقم القياسى التجميعي بالطرق المختلفة لأهم محاصيل الحبوب المختلفة

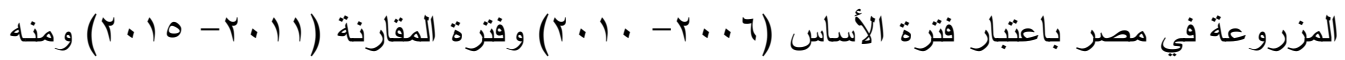
يتبين أن رقم فيشر القياسى يحتل مكانة أقل من الأرقام القياسية التي تم تقدير ها بأساليب مختلفة فضراًاً

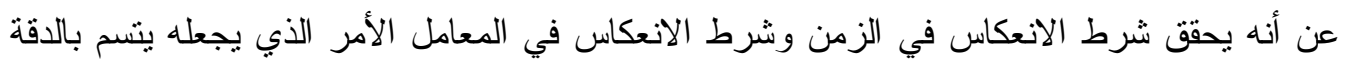
و الجودة عن غيرهـ من الأرقام القياسية.

'همسة معن محمد ثابت (T/ • ب)، "استحداث خوارزمية جينية لاختبار أمتلية الأرقام القياسية"، جامعة الموصل المجـــة

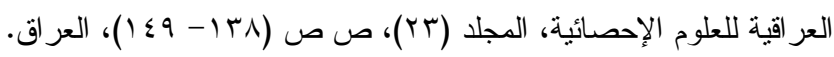


شكل (1) تقدير الرقم القياسى التجميعي لأهم المحاصيل الحبوب بالطرق المختلفة خلا فترة

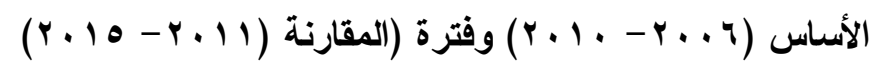

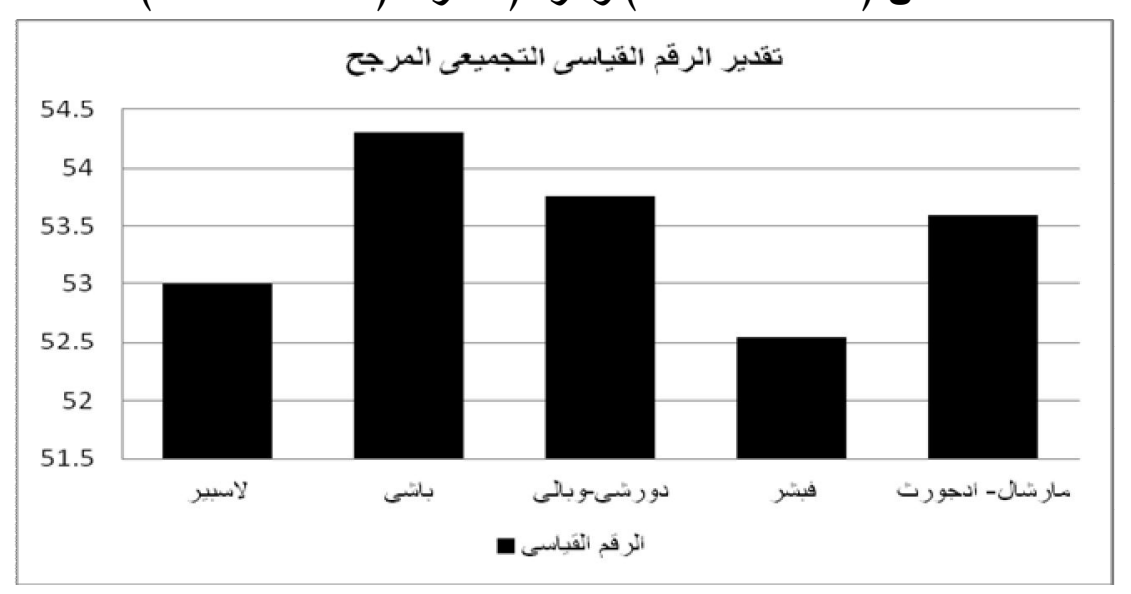

أهمية الرقم القياسى فى تقدير التضخم

التضخم هو الزيادة فى الطلب الكلى على العرض الكلى زيادة محسوسة تؤدى إلى سلسلة

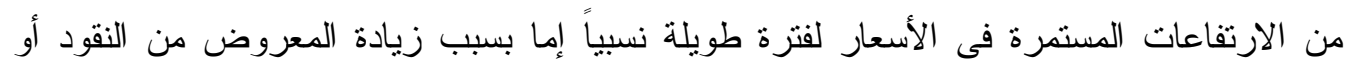

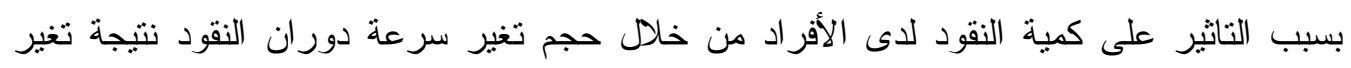
عادات الثر اء عند الأفر اد مما يؤدى فى النهاية إلى الارتفاعات المستمرة فى الأسعار بدرجة كبيرة

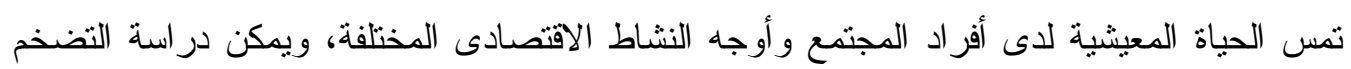

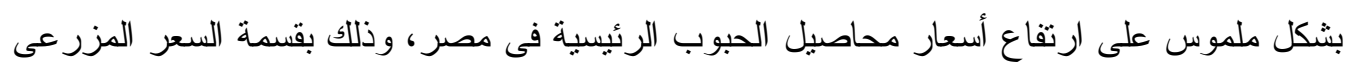

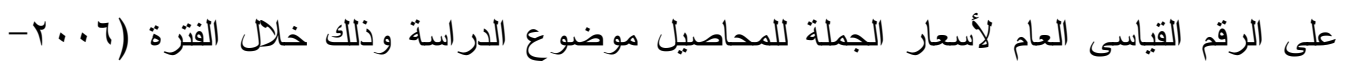

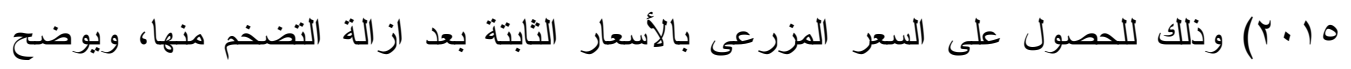

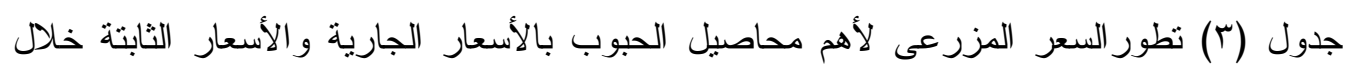

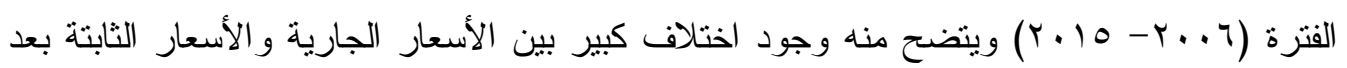

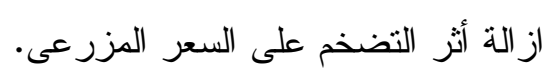

جدول (r) تطور الأسعار المزرعية الجارية والثابتة بالجنيه لأهم محاصيل الحبوب في مصر

\begin{tabular}{|c|c|c|c|c|c|c|c|c|c|c|}
\hline \multicolumn{2}{|c|}{ أسعار الشعير } & \multicolumn{2}{|c|}{ أسعار القمح } & \multicolumn{2}{|c|}{ أسعار الأرة الرفيعة } & \multicolumn{2}{|c|}{ أسعار الأرة الثُامية } & \multicolumn{2}{|c|}{ أسعار الأزز } & \multirow{2}{*}{ السنة } \\
\hline ثُابت & جاري & تابت & جاري & تابت & جاري & ثابت & جاري & ثابت & جاري & \\
\hline$\lambda T V$ & 1.90 & 9.9 & $11 Y V$ & $\lambda q$. & $11 . \varepsilon$ & $\lambda V$. & 1.199 & 179 & $1 . V V$ & Y..T \\
\hline 9.7 & $1 r \leqslant r$ & $\Lambda \leqslant Y$ & 1104 & $\mid Y \cdot\{$ & 170. & $110 \mathrm{r}$ & $10 V 9$ & 1.09 & $1 \leqslant 01$ & $r \ldots V$ \\
\hline $10 \leqslant 0$ & rIIV & 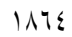 & roor & $1 . \leqslant 1$ & $1 \leqslant \mu$ & $1 . r r$ & $\mid\{1\}$ & 1.79 & $1 \leqslant 70$ & $r \ldots \lambda$ \\
\hline ATr & $r T \leqslant Y$ & 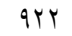 & 1714 & ANTV & $1 \leq 7 \leq$ & VAA & $1 \% V q$ & $10 \leqslant$ & $1 \leqslant 90$ & $r \ldots q$ \\
\hline $1 \times 97$ & $r \leqslant V_{0}$ & $9 \vee 9$ & $1 \mathrm{NIT}$ & 991 & 1194 & $9 \Lambda$. & $|A V|$ & 974 & IATV & r.l. \\
\hline ITY & rort & Irra & $r r \leqslant V$ & $1 . \mathrm{rA}$ & 1978 & 1.1. & $19 r 9$ & 1.01 & $r \ldots \Lambda$ & r.11 \\
\hline IVTr & rrat & . & rory & 1194 & MYTE & 11149 & YIT\& & 1.11 & T.TV & r. Ir \\
\hline 1194 & $r \leqslant 0 \Lambda$ & 19. & ros. & $\vee 99$ & 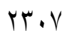 & VVT & rTS & VYA & ril. & $r .1 T$ \\
\hline $17 \leqslant \leqslant$ & ra.. & 1501 & $r V \leqslant$. & $1 . \mathrm{V}$. & $r r \leqslant r$ & $1 . r \varepsilon$ & MYYS & 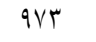 & rir. & T.1 \\
\hline IVo. & s.ro & $119 \mathrm{~V}$ & ryor & $1 . Y \Lambda$ & Fו" & $1 \ldots$ & rT... & $q r q$ & rluy & $r .10$ \\
\hline
\end{tabular}

المصدر: وزارة الزراعة واستصلاح الأراضي، قطاع الشئون الإتصادية الإدارة المركزية للاقتصاد الزراعي، نشرة الإحصاءات الزراعية، أعداد مختلفة. 


\section{معادلات الاتجاه الزمني لتطور السعر المزرعى}

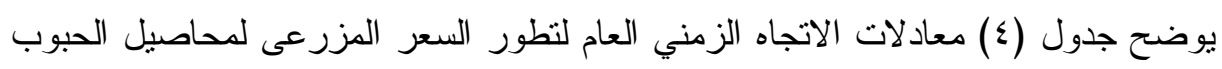

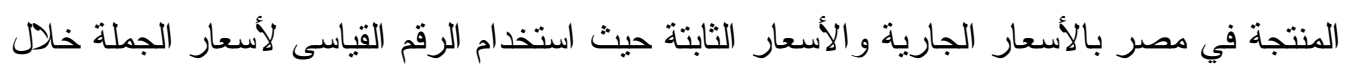

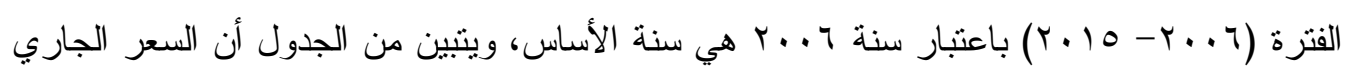

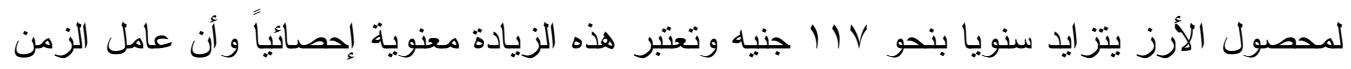

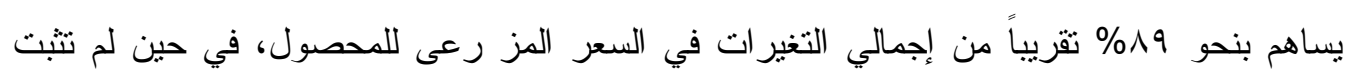

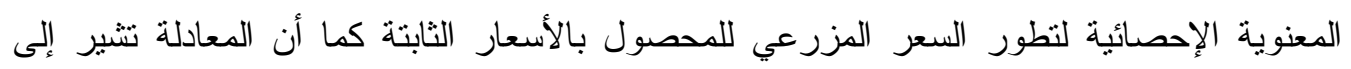
الانخفاض في السعر المزرعى للمحصول، وليس زيادة كما في حالة الأسعار الجارية.

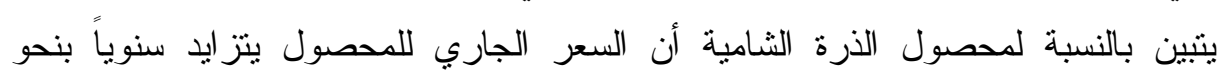

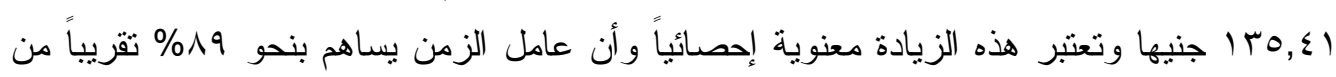

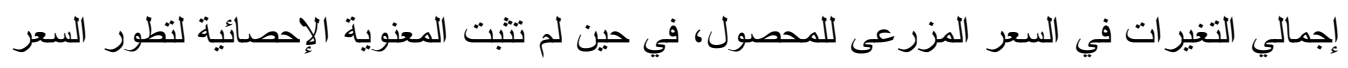

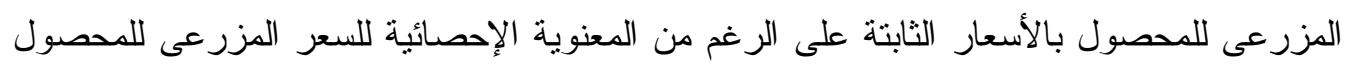

$$
\text { بالأسعار الجارية. }
$$

تشير معادلة الاتجاه الزمني العام لتطور السعر الجاري لمحصول الذرة الرفيعة خلال الفترة

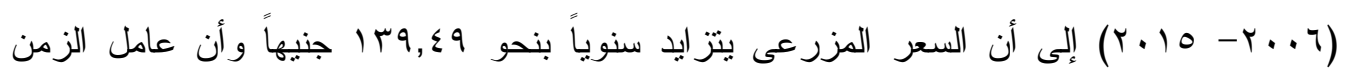

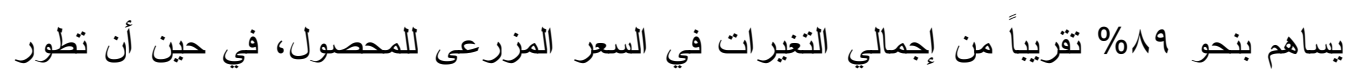
السعر الثابت للمحصول ينز ايد سنوياً بمعدل معنوي إحصائياً.

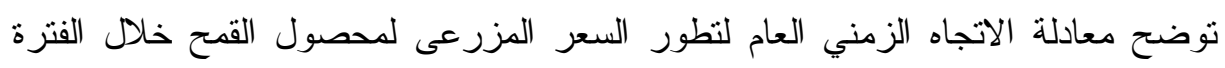

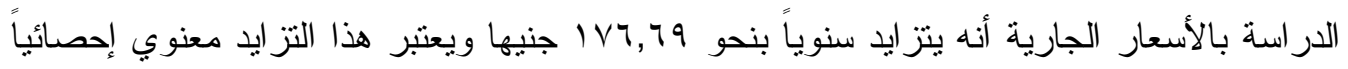

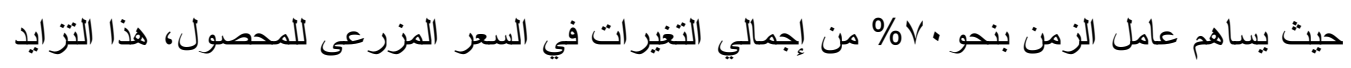

$$
\text { لم يثبت معنونته الإحصائية. }
$$

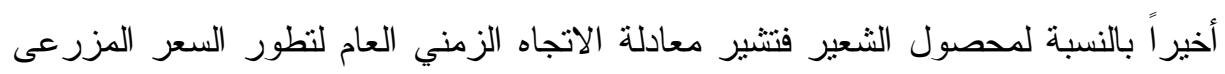

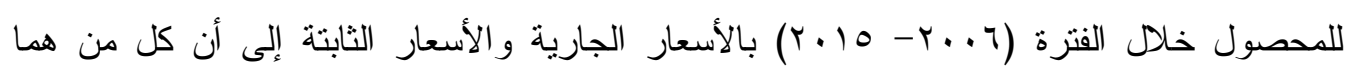

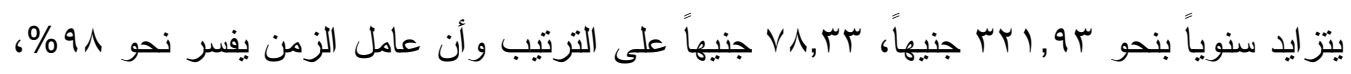

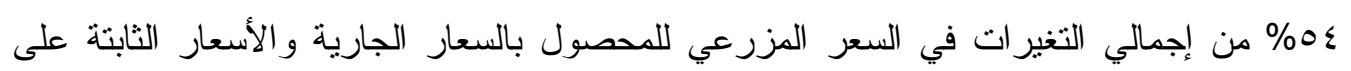


جدول (ء) معادلات الاتجاه الزمني العام لتطور أسعار محاصيل الحبوب

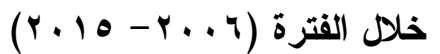

\begin{tabular}{|c|c|c|c|c|c|}
\hline المغنوية & $\boldsymbol{F}$ & $R^{2}$ & المعادلة & بالأسعار & المحاصيل \\
\hline$* *$ & 65.82 & 0.89 & $\hat{Y}_{i}=1131.01+{ }_{(12.58)^{88}}^{117.511)^{* *}} X_{i}$ & الجارية & \multirow[t]{2}{*}{ الأرز } \\
\hline- & 0.196 & 0.024 & 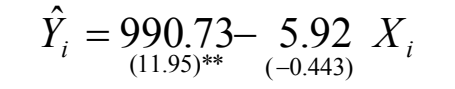 & الثابتة & \\
\hline$* *$ & 62.67 & 0.89 & $\hat{Y}_{i}=\underset{(10.152)^{* *}}{1077.47}+\underset{(7.928)^{* *}}{135.41 X_{i}}$ & الجارية & \multirow{2}{*}{ الذرة الثشامية } \\
\hline- & 0.003 & 0.00 & $\hat{Y}_{i}=\underset{(10.29)^{* *}}{973.4}+\underset{(0.052)}{0.800}$ & الثابتة & \\
\hline$* *$ & 62.5 & 0.89 & $\hat{Y}_{i}=11_{(10.15)^{* *}}^{111.67+139.49} X_{(7.91)} X_{i}$ & الجارية & \multirow{2}{*}{ الذرة الرفيعة } \\
\hline- & 0.003 & 0.00 & $\hat{Y}_{i}=\underset{(98.6)^{* * *}}{1003.5}+\underset{(15.9)}{0.885} X_{i}$ & الثابتة & \\
\hline$*$ & 14.76 & 0.70 & $\hat{Y}_{i}=1_{(4.53)^{* *}}^{1148.8}+\underset{(4.33)^{* *}}{176.69} X_{i}$ & الجارية & \multirow{2}{*}{ القمح } \\
\hline- & 0.123 & 0.02 & $\hat{Y}_{i}=\underset{(4.77)^{* *}}{1068.67}+\underset{(0.351)^{*}}{12.66} X_{i}$ & الثابتة & \\
\hline$* *$ & 201.9 & 0.98 & $\hat{Y}_{i}=\underset{(0.997)^{* *}}{840.24}+\underset{(14.21)^{* *}}{321.93} X_{i}$ & الجارية & \multirow[t]{2}{*}{ الشعير } \\
\hline$*$ & 9.579 & 0.54 & $\hat{Y}_{i}=\underset{(5.89)^{* * *}}{924.87}+\underset{(3.09)^{*}}{78.33} X_{i}$ & الثابتة & \\
\hline
\end{tabular}

المصدر : جمعت وحسبت من بيانات وزارة الزر اعة واستصلاح الأر اضي، قطاع الثئون الاقتصادية، نشرة الإحصاءات

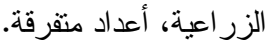

مما سبق يتبين أن استخدام الرقم القياسي في تقدير أسعار الجملة بعد استبعاد أثر التضخم

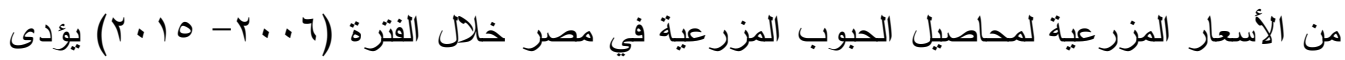
بدرجة ملحوظة إلى اختلاف معنوية معدل النطور الزمني لأسعار محاصيل الحبوب واختلاف معنوية

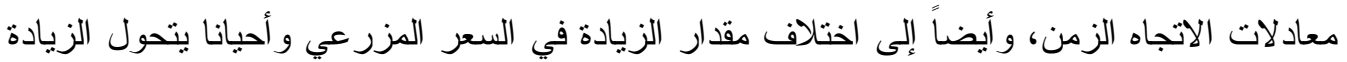

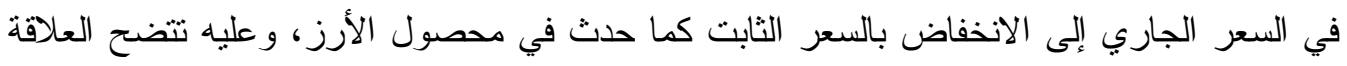
العكسية بين المستوى العام للأسعار المزرعية محاصيل الحبوب وقيمة النقود والتي غالباً ما تتأثر

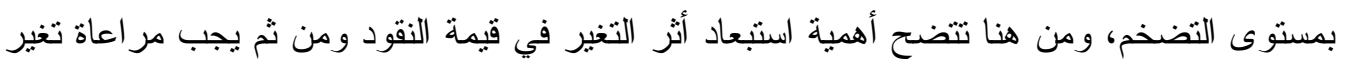

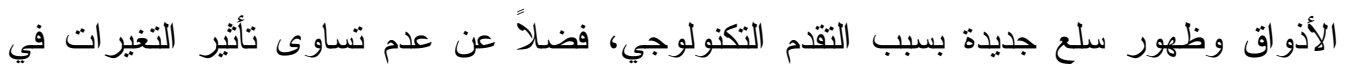
مستويات الأسعار لكافة أفر اد المجتمع و الذي يعنى أن هناك فئة قد تتأثز دون فئة أخرى. أيضاً اختلاف أذو اق استهلاك محاصيل الحبوب بين الريف و الحضر ومن منطقة إلى أخرى، و وأن

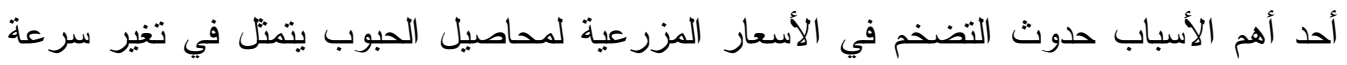

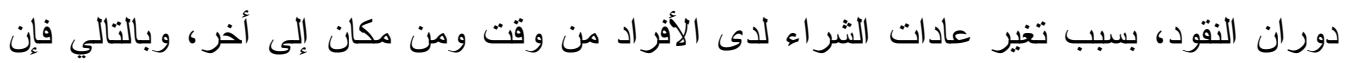

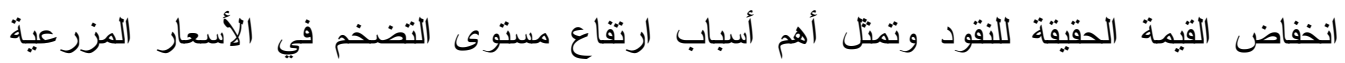
لمحاصيل الحبوب وما يترتب عليها من ارتفاع تقدير الرقم القياسي العام لأسعار الجملة. 


\section{مراجع باللغة العربية}

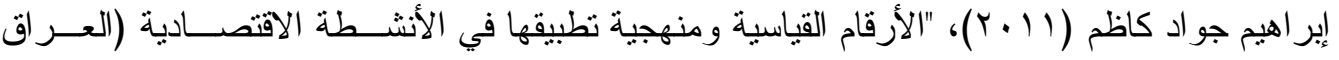

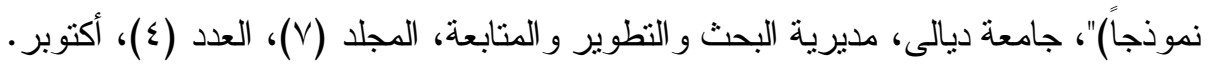

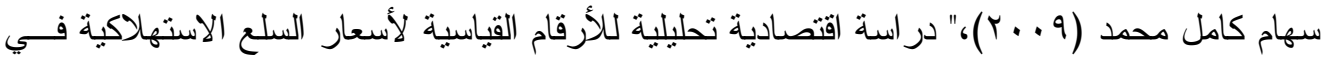

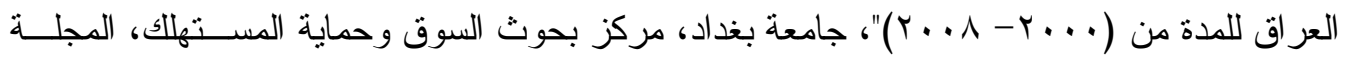
العر اقية لبحوث السوق وحماية المستهلك، المجلد (1)، العدد (r). صالح العصفور (T . . Y)، "الأرقام القياسية"، المعهد العربي للتخطيط بالكويت، العدد (9 ())، يوليو.

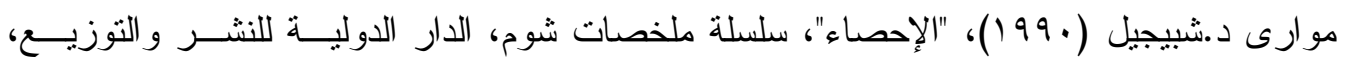

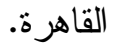

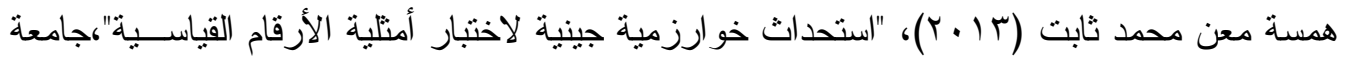

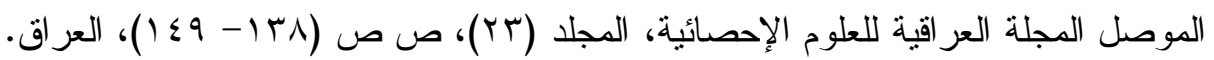

\section{مر اجع باللغة الانجليزية}

Newmark J. (1991)," Statistics and probability in modern life", the college of Staten island of the city university of new York, fifth edition

$$
\text { النشرات والدوريات }
$$

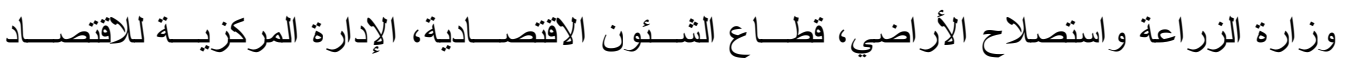
الزر اعي، نشرة الإحصاءات الزر اعية، أعداد مختلفة. 


\section{الملحق}

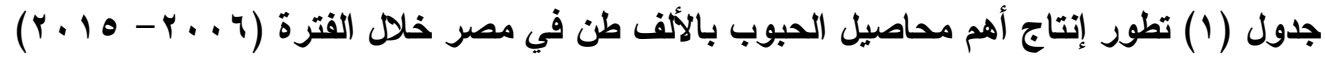

\begin{tabular}{|c|c|c|c|c|c|}
\hline الثعير & القمح & الأرة الرفيعة & الأرة الشامية & الأرز & السنوات \\
\hline IT & $A Y V \varepsilon, r$ & NVT & 710. & $T V \varepsilon \varepsilon, Y$ & $r \ldots r$ \\
\hline $\mid r v, 1$ & $\vee r \vee \wedge, q$ & VAo & 7117 & $\neg \wedge \neg \wedge, r$ & $r \ldots r$ \\
\hline . & $\vee 9 \vee \vee, 1$ & 101 & Tr. & $V Y \leqslant \cdot, 0$ & $r \ldots \lambda$ \\
\hline $1 \leq 7, r$ & NOrT & $V \vee \varepsilon$ & $7 \nearrow \leq \varepsilon$ & 0011,1 & $r \ldots q$ \\
\hline 118,1 & 1179 & $79 r$ & TYVT & $\varepsilon r r v, 1$ & $r \cdot 1$. \\
\hline Kr, & $\Lambda r V \cdot, 0$ & NTI & $0 \wedge \wedge 7$ & 0770,5 & $r .11$ \\
\hline $1 \cdot v, 7$ & $\Lambda \vee 90,0$ & vol & $V Y \cdot T$ & $0 \wedge 97,7$ & $r .1 r$ \\
\hline r, & $9 \leq 7 \cdot, r$ & vo9 & $V 1 \cdot r$ & ovIV, I & $r .1 T$ \\
\hline $1 \cdot 1$, & $9 Y \vee q, \wedge$ & $1 \cdot 1$ & $V Y \leq T$ & $0 \leqslant 7,, V$ & $r \cdot 1 \leq$ \\
\hline$\cdots, v$ & $97 \cdot V, V$ & 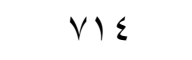 & $v .01$ & $\varepsilon \wedge \mid \wedge$ & $r .10$ \\
\hline
\end{tabular}

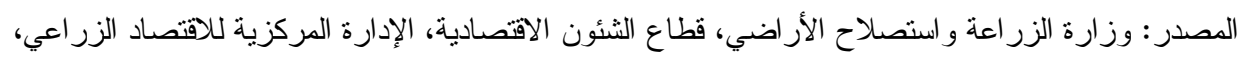
نشرة الإحصاءات الزر اعية، أعداد مختلفة.

جدول (ץ) تطور الأسعار المزرعية بالجنيه لأهم محاصيل الحبوب في مصر

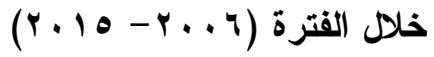

\begin{tabular}{|c|c|c|c|c|c|}
\hline الثعير & القمح & الذرة الرفيعة & الثامية & الأرز & السنوات \\
\hline $1 . \mathrm{ro}^{\prime}$ & $11 r V$ & $11 . \varepsilon$ & 1.19 & $1 . V V$ & $r \ldots r$ \\
\hline ITEY & 1104 & 170 & $10 V 9$ & $1 \leqslant 01$ & $r \ldots r$ \\
\hline rIIV & roor & $1 \leqslant \pi$ & $|\leqslant| \varepsilon$ & $1 \leq 70$ & $r \ldots \lambda$ \\
\hline$r \quad r \leqslant r$ & Tו17 & $1 \leq 7 \leq$ & irva & $1 \leqslant 90$ & $r \ldots q$ \\
\hline$r \leqslant V_{0}$ & NMT & 1199 & $|A v|$ & IATV & $r .1$. \\
\hline rotr & $r r \leqslant V$ & $197 \varepsilon$ & $19 r 9$ & $r \ldots \wedge$ & $r+11$ \\
\hline rrqT & rorv & TrTE & YTY & $r \cdot T V$ & $r .1 r$ \\
\hline$r \leqslant 0 \wedge$ & ron. & $r r \cdot v$ & $r \quad\{r$ & rul. & $r .1 r$ \\
\hline r... & $r V \leq$. & $r \quad r \leqslant r$ & TYTE & rir. & $r \cdot 1 \varepsilon$ \\
\hline E.ro & ryor & ETY & rr.. & צrוז & $r .10$ \\
\hline
\end{tabular}

المصدر: وزارة الزر اعة واستصلاح الأراضي، قطاع الثئون الاقتصادية، الإدارة المركزية للاقتصاد الزر اعي، نشرة الإحصاءات الزراعية، أعداد مختلفة. 
